\title{
When Hedge Funds Block the Exits
}

\author{
Andrew Ang* \\ Columbia Business School
}

Nicolas P.B. Bollen

Vanderbilt University

\begin{abstract}
The ability of hedge fund investors to exit a fund by exchanging ownership for cash at the prevailing NAV is often blocked by lockups and notice periods. We model the exit decision as a real option and incorporate lockups and notice periods as exercise restrictions. We compute the cost of these restrictions using a lattice that incorporates the possibility of fund failure. Using data through 2008, we estimate that a two-year lockup with a three-month notice period costs approximately $4 \%$ of the initial investment. The cost of illiquidity can easily exceed $5 \%$ per year if the hedge fund manager suspends withdrawals as was common in the months following the financial crisis.
\end{abstract}

January 14, 2010

*Andrew Ang, phone: (212) 854-9154, email: aa610@columbia.edu, Nicolas Bollen, phone: (615) 3435029, email: nick.bollen@ owen.vanderbilt.edu. The authors thank Emanuel Derman, Greg van Inwegen, Francis Longstaff, Hans Stoll, Jacob Sagi, and Neng Wang; seminar participants at Cornell University, the University of Mississippi, Vanderbilt University, and Virginia Tech; as well as attendees of the $1^{\text {st }}$ Annual Conference on the Econometrics of Hedge Funds (Paris) and the $3^{\text {rd }}$ Conference on Professional Asset Management (Rotterdam) for helpful comments. Support from the Financial Markets Research Center is gratefully acknowledged. 


\section{When Hedge Funds Block the Exits}

\section{Introduction}

Many alternative investment vehicles place restrictions on the ability of investors to redeem capital. A lockup requires an investor to wait a specified length of time, typically one to three years, before requesting a redemption. A notice period requires an investor to wait a specified length of time, typically one to three months, before a redemption request is processed. In addition, gates allow fund managers to process an arbitrary fraction of a redemption request or even to suspend redemptions altogether.

Redemption restrictions allow fund managers to invest in illiquid assets and earn associated return premiums for the benefit of fund investors. However, redemption restrictions also levy a substantial cost by preventing investors from withdrawing capital when a fund underperforms. As markets collapsed in 2008, for example, many fund managers barred withdrawals and maintained restrictions through 2009. ${ }^{1}$ This paper demonstrates how investors can compute the cost of restrictions ex-ante in order to make informed investment decisions. We model the exit decision as a real "liquidity" option and treat lockups and notice periods as exercise restrictions.

Our approach has three key elements. First, our data generating process for hedge fund returns has two components: a normal regime when the fund is alive and an absorbing failure state in which investors are forced to accept a payout per share equal to a fraction of the fund's NAV. We employ a log-logistic duration function to predict hedge fund failure and allow hazard rates to depend on realized performance. Thus, the cost of redemption restrictions changes over time as a function of fund age and performance: restrictions become more expensive for a fund with poor cumulative performance since it is more likely to fail.

Second, for the cost of lockups and notice periods to be meaningful, there must be periods where it is optimal for an investor to withdraw capital and receive the NAV, i.e. there is a difference between a fund's NAV and an investor's valuation of ownership in the fund. ${ }^{2}$ In our setting an investor's valuation of ownership in the fund differs from the NAV because the NAV reflects neither the capitalization of future managerial performance nor the probability of fund failure and the associated liquidation cost.

Third, we value an investor's liquidity option, and the compute the cost of redemption restrictions, using a binomial lattice that embeds time-varying probabilities of fund failure and, most importantly, allows for early exercise. Upon exercise the investor exchanges ownership in the fund for the NAV. We measure the cost of a lockup as the difference between the value of a liquidity option that allows exercise at any time and another that does not permit exercise during the lockup period. We measure the cost of a

\footnotetext{
${ }^{1}$ See “Hedge Fund Investor Goal: An Exit Plan,” Wall Street Journal, September 9, 2009.

${ }^{2}$ If the fund is invested in illiquid assets for which market prices are not readily available, the fund manager may employ subjective marking to model when computing the fund's NAV, and the NAV may be susceptible to managerial misreporting. See, for example, Asness, Liew and Krail (2001), Getmansky, Lo and Makarov (2004), and Bollen and Pool (2008). We do not incorporate differences of opinion regarding the value of the fund's portfolio.
} 
notice period as the difference between the value of a liquidity option that provides an immediate payoff equal to the NAV and the value of a liquidity option that requires a waiting period before the payoff is determined. We also compute the combined cost of lockups and notice periods.

We estimate the cost of lockups and notice periods by calibrating our model to a large sample of hedge funds using the CISDM and TASS databases. Using a standard set of fund attributes, we estimate that the combined cost of a two-year lockup and a threemonth notice period is approximately $4 \%$ of the initial investment for a power-utility investor with risk aversion level of $3 .{ }^{3}$ More risk-averse investors assign higher costs to lockups and notice periods because they tend to want to liquidate sooner and the exercise restrictions are binding more often. A manager's discretion to block redemption requests using gates generates an implied cost that can easily exceed 5\% per year assuming that an investor initially values the fund investment at par. These results suggest that in some cases the illiquidity premiums that are earned by investors' captive capital may be insufficient to offset the cost of restrictions on the ability to exit a fund.

\section{Related Research}

Our paper is related to existing studies of the relation between redemption restrictions and hedge fund returns. Ding et al. (2007) show that redemption restrictions affect the empirical cross-sectional relation between aggregate capital flow and returns. Aragon (2007) documents that hedge funds with lockups have expected returns that are $4 \%-7 \%$ per annum higher than hedge funds without lockups. Aragon interprets this difference as an illiquidity premium: lockups allow managers to invest in more illiquid securities and earn higher returns as a result. However, Aragon does not explicitly compute the cost of a lockup and cannot determine if the illiquidity premium is fair compensation. A related literature explores whether lockup provisions are a component of an optimal incentive contract for a fund manager, especially one investing in illiquid assets, as in Lerner and Schoar (2004).

Our paper is most related to Derman (2007) who models hedge fund returns using a three-state model in which there are three types of hedge funds: good, sick, and dead. Derman, Park and Whitt (2007) extend this approach to allow for more complex Markov chain models. In both approaches, lockups prevent an investor from withdrawing capital from a sick fund and investing the proceeds in a good fund. Our valuation strategy differs from Derman (2007) in four important ways. First, Derman assumes investors swap capital invested in a poorly performing hedge fund for capital invested in a superior hedge fund, whereas we assume investors withdraw capital as cash. Thus, our approach explicitly models the actual decision that investors face. Second, we take into account the risk aversion of an investor. Third, we differentiate between lockups and notice periods, and develop a methodology that can estimate the cost of the two restrictions separately, or in combination. Finally, we model fund failure using a hazard rate that can depend on fund age and performance, as described next.

\footnotetext{
${ }^{3}$ This level of risk aversion corresponds to a $60 \%-40 \%$ equity-bond asset allocation mix for an investor holding only U.S. equities and risk-free bonds.
} 


\section{Methodological Setup}

\subsection{Modeling fund NAVs}

We assume that continuously compounded fund NAV returns are initially normally distributed and that this "normal regime" continues as long as the fund survives. We use a binomial lattice to model the evolution of a hedge fund's NAV. Let $S_{t, j}$ denote the NAV, where $t$ denotes the time step, running from 0 to $T$, and $j$ denotes the level in the lattice, running from 1 to $t+1$ at time step $t$, with 1 being the highest. We refer to the combination of time step $t$ and level $j$ as node $(t, j)$. The time between nodes is denoted by $\Delta t$. Date $T$ represents the end of the hedge fund's life if failure never occurs. This can be interpreted as either the investor's investment horizon, the retirement of the hedge fund manager, or the feasible horizon of the fund's investment strategy, and the purposeful unwinding of the hedge fund's positions.

The lattice is defined by the step size $u$ and branch probability $p$, which are determined setting the mean and variance implied by the lattice equal to those of the hedge fund's normal regime. With probability $p$ the NAV increases from $S_{t, j}$ to $S_{t+1, j}$ where

$$
S_{t+1, j}=S_{t, j} u,
$$

with the multiplicative increase $u$ and the probability $p$ given by

$$
\begin{aligned}
& u=e^{\sigma \sqrt{\Delta t}} \\
& p=\frac{e^{\mu \Delta t}-u^{-1}}{u-u^{-1}},
\end{aligned}
$$

where $\mu$ and $\sigma$ are the mean and standard deviation of hedge fund returns in the normal regime. With probability $1-p$ the NAV decreases from $S_{t, j}$ to $S_{t+1, j+1}$ where

$$
S_{t+1, j+1}=S_{t, j} u^{-1}
$$

\subsection{Failure process}

Let $D$ be the duration of a hedge fund, which we define as the random time that a fund fails, and at which point the manager liquidates the fund's remaining assets. Empirically, we measure duration as the time that a hedge fund manager stops reporting returns. While some hedge fund managers may stop reporting for good performance, the majority of funds cease reporting due to failure as argued by Ackermann, McEnally and Ravenscraft (1999) and Grecu, Malkiel and Saha (2006). If the fund fails at node $(t, j)$, we assume that the fund NAV drops to a level $S_{t, j} l$ where $l$ represents the proportion of pre-failure NAV that the manager is able to raise through liquidating asset sales, with $0<l<1$. The investor receives the liquidating dividend at time $t+1$. 
Upon failure, we assume as a base case that investors receive a payoff of $l=75 \%$ of the prevailing NAV of the fund, reflecting additional loss of asset value during liquidation. The $25 \%$ liquidation cost is based on results reported in Ramadorai (2008), who analyzes a sample of transactions on a secondary market for hedge fund investments conducted on Hedgebay. During 66 "disaster" transactions, involving fraud or collapse, the average discount of transaction price to NAV is $49.6 \%$. To be conservative, we halve Ramadorai's estimate. ${ }^{4}$

We follow Grecu, Malkiel and Saha (2006) and use the log-logistic distribution as a model for hedge fund durations as they show this model fits the empirical density of hedge fund durations better than other distributions. This hazard rate is given by:

$$
\lambda_{b}(t)=\lambda q(\lambda t)^{q-1} /\left[1+(\lambda t)^{q}\right] .
$$

In addition to this baseline hazard rate, we also assume that failure rates can be shifted up and down depending on hedge fund cumulative performance relative to other funds. Specifically, the hazard rate of an individual hedge fund equals the baseline hazard rate scaled up or down depending on the value of covariate $z$ as follows:

$$
\lambda(t ; z)=\lambda_{b}(t) e^{z \beta}
$$

We follow standard practice and demean the covariate so that values above or below zero increase or decrease the hazard rate. We choose a performance-based covariate equal to the difference between the cumulative return of fund $i$ at time $t$ and the cross-sectional mean return. The difference is then scaled by the cross-sectional standard deviation. The cross-sectional mean and standard deviation are computed using the cumulative returns of each fund when they are the same age as fund $i$ at time $t$.

The sensitivity of the failure rate to performance makes the hazard rates vary over time and is important to include for three reasons. First, and most importantly, Liang (2000), Brown, Goetzmann and Park (2001), and Jagannathan, Malakhov and Novikov (2006), among others, empirically document that liquidated hedge funds are more likely to be funds with poor past performance. Second, managers of funds with low cumulative returns are less likely to capture performance fees, since the NAV must recover to previously set high-water marks before the fees accrue. This provides managers of poorly performing funds a strong incentive to close those funds. Third, investors are more likely to withdraw capital from poorly performing funds, forcing the manager to liquidate assets, possibly leading to further reductions in NAV, again leading the manager to close those funds. In other models of hedge fund failure, like the model used by Derman, Park and Whitt (2007), failure probabilities are constant.

We estimate the hazard rate parameters in (4) and (5) using hedge fund data from the Center for International Securities and Derivatives Markets (CISDM) and TASS

\footnotetext{
${ }^{4}$ In conversations with fund-of-funds managers, our value of the NAV dropping $25 \%$ contingent upon failure was remarked to be conservative, with their (informal) estimates closer to $50 \%$. Some failing funds have a drop of nearly $100 \%$ in value contingent upon default, even in cases not involving fraud. For example, investors in Citigroup's Corporate Special Opportunities Fund received 3 cents on the dollar when the fund was wound up, as reported in "Investors Hammered by Citi Fund Setback," Financial Times, January 15, 2009.
} 
databases. The sample period runs through December 2008. We extract from these databases all live and defunct individual hedge funds.

Table 1 lists parameter estimates of the hazard rate function in (4) and (5). Parameters of the baseline log-logistic function in (4) are $\lambda=0.0164$ and $q=3.1539$, both estimated very precisely. The implied duration at which the unconditional probability of survival is $50 \%$ equals approximately 60 months. Figure 1 compares the actual number of hedge funds with uncensored duration $t$ to the predicted number based on the parameter estimates of $\lambda$ and $q$. The predicted number equals the hazard rate evaluated at duration $t$ times the total number of funds, both live and defunct, with history length at least $t$. The fit is good and suggests that fund age is a significant determinant of the probability that a manager will stop reporting to the database. The other determinant in our specification is the relative cumulative fund performance which shifts the baseline hazard rate up or down. Table 1 reports the maximum likelihood estimate of $\beta$ is -0.2302 with standard error 0.0057 . Thus, a cumulative return that is one standard deviation below the mean increases the hazard rate by about $21 \%$.

The hazard rate in (5) allows for the probability of failure to depend non-linearly on age and realized performance of the fund. In many option applications state-dependent payoffs lead to path dependence and cause the number of nodes in a lattice to explode. A major advantage of our approach is that we specify the performance covariate so that it can be computed at each node in the lattice without knowledge of the path taken.

Let $\pi_{t, j}$ denote the probability of failure at node $(t, j)$, with the failure occurring prior to the return of the fund being realized between time $t$ and $t+1$. At node $(t, j)$ we numerically evaluate this by computing the hazard rate halfway between time $t$ and $t+1$, i.e. we set $\pi_{t, j}=\lambda_{b}\left(\operatorname{age}_{t}+0.5\right) \Delta t$ in the case of the base hazard rate and $\pi_{t, j}=\lambda_{b}\left(\right.$ age $\left._{t}+0.5\right) e^{z_{t, j} \beta} \Delta t$ in the case of the proportional hazard rate, where $\Delta t$ is the increment of time in the lattice, $z_{t, j}$ is the value of the performance covariate at node $(t, j)$ , and age $e_{t}$ is the age of the fund at time $t$. The hazard rates over $t$ to $t+\Delta t$ depend on the value of the covariate at $t$. Note that time $t=0$ corresponds to the investor's initial subscription to the fund rather than the fund's age.

\section{Valuing Liquidity Options and Lockups}

We now briefly describe a procedure for valuing a hedge fund, the investor's option to withdraw capital, and the cost of a lockup. Our procedure can be extended to value notice periods using sub-trees as described in Ang and Bollen (2010). We assume that investors are risk-averse with a power utility function for time $T$ wealth, $W_{T}$, given by

$$
U\left(W_{T}\right)=W_{T}^{1-\gamma} /(1-\gamma),
$$


where $\gamma$ is the agent's risk aversion. ${ }^{5}$ We expect, and confirm below, that more riskaverse investors assign a higher cost to redemption restrictions than less risk-averse investors.

One way to interpret the risk aversion parameter $\gamma$ is to consider the equity-bond asset allocation implied for a power utility investor restricted to holding only equities and risk-free bonds. Using Ibottson data from January 1926 to December 2008, a $\gamma=3$ investor would hold a 55\%-45\% equity-bond mix assuming all asset returns are lognormally distributed. A $60 \%-40 \%$ equity-bond allocation corresponds to a risk aversion of $\gamma=2.7$.

The intuition behind our methodology for valuing lockups is as follows. We compute the value of a hedge fund per share where there are no restrictions on converting the NAV to cash. We denote this value by $O$. In certain states, like when default is imminent, the investor wishes to exercise the liquidity option because the value received in cash (the NAV) is greater than the expected value of the fund next period. A share subject to a lockup restriction cannot be exercised during the lockup period and we denote its value by $O_{L}$. Since the lockup restriction prevents an investor from maximizing value during the lockup period, the restricted share is worth less than the unrestricted share. The cost of the lockup, therefore, is the difference between the restricted and unrestricted shares, $O-O_{L}$. We denote the case where the manager always restricts share redemptions - unfortunately these are precisely the times when an investor would most want to receive cash - as $H$. The difference $O-H$ can be considered to be the cost of gate provisions where the manager always restricts redemptions when exercising is advantageous to the investor.

The valuation of the hedge fund values with liquidity restrictions, $O_{L}$ and $H$, and the unrestricted hedge fund value without any liquidity restrictions, $O$, are computed using the binomial tree. The valuation method is similar to the computation of American options with three differences. First, utility certainty equivalents are used because in general risk-neutral valuation is not possible as the underlying hedge fund cannot be shorted. Second, the tree represents the hedge fund returns conditional on no default occurring. Once the hedge fund fails, which has time-varying probabilities dependent on past hedge fund returns, the liquidation value is received. Third, the American exercise feature where the investor desires the cash proceeds of the fund is disabled during the lockup period. The appendix contains details on the underlying methodology.

\section{Costs of Lockups and Notice Periods}

\subsection{Costs Assuming Investment in the Fund is Already Made}

Table 2 lists the combined costs of lockups and notice periods when the initial fund NAV is $\$ 100$, returns are normally distributed with annual expected return of $10 \%$ and volatility of $15 \%$, the fund has a 10-year horizon, fund failures incur a $25 \%$ loss upon liquidation, and the risk-free rate is $2 \%$ per annum. Funds have age equal to 24 months at

\footnotetext{
${ }^{5}$ The power utility function is also called Constant Relative Risk Aversion or CRRA utility.
} 
$t=0$ and we specify risk aversion $\gamma=3$. Costs are increasing in the length of the lockup and notice period. For a notice period of three months, the combined cost of restrictions ranges from $\$ 1.03$ for a one-year lockup to $\$ 10.58$ for a five-year lockup. For a lockup of three years, the combined cost of restrictions ranges from $\$ 6.62$ for a one-month notice period to $\$ 7.49$ for a five-month notice period. These results indicate that the length of the lockup has much more impact on the combined cost of restrictions than the length of the notice period. Introducing notice periods has only a small effect on hedge fund value because the probability of failure during the notice period is relatively small.

\subsection{Costs Assuming Investment in a New Fund}

So far we have computed the costs of notice periods and lockups assuming that investment in the hedge fund has already been made. An underlying assumption is that the liquidity options have value because the NAV of the fund does not capture all the relevant valuation variables, especially termination probabilities and liquidation costs. In this section we assume that at initiation the investor is indifferent between investing and not investing in the fund. That is, the initial NAV is the fair value to the investor and incorporates the probability of all aspects of hedge fund failure.

We calibrate our numerical examples so that the investor's valuation of the hedge fund value assuming an unrestricted liquidity option exactly equals the initial NAV of $\$ 100$. We achieve this by selecting appropriate combinations of expected returns and volatilities as listed in Table 3. Each row of Table 3 corresponds to a different

combination of $\mu$ and $\sigma$. By construction the value of $O_{0,1}$ for all rows is 100 . Next, we add restrictions on the liquidity option. Naturally this reduces the value of the hedge fund, so we compute the incremental required return per annum to compensate the investor for bearing each additional restriction, raising the hedge fund value back to par.

Consider the lowest level of $\mu$ and $\sigma$ in Table 3. Starting from the right-most column, the imposition of a three-month notice period requires an additional $0.28 \%$ expected return per year for the investor to be compensated. In contrast, a two-year lockup requires an additional $3.20 \%$ per year. Again in this example lockups are significantly more costly than notice periods. When the liquidity option is completely eliminated, the necessary increase in expected return to adequately compensate the investor is $5.58 \%$ per year. All these liquidity costs become larger as volatility increases.

In summary, for an investor who disregards liquidity restrictions and initially values the fund fairly at par, elimination of the ability to redeem is equivalent to an expected return shortfall of over $5 \%$ per year. This result illustrates the tremendous cost imposed on investors when managers exercise their option to prohibit investor withdrawals.

\subsection{Restriction Costs at January 2009}

The results described above are for hypothetical funds with an assumed age of two years and representative return attributes. How big are restriction costs in practice? To answer this question, we study all live hedge funds in the CISDM and TASS 
databases at the beginning of 2009 using their historical returns through December 2008. For each fund we record its age, average return, and volatility at the start of January 2009 and then compute the cost of liquidity restrictions using our binomial procedure. For each fund we compute the costs of three sets of restrictions: "Short" corresponds to a onemonth notice period and a one-year lockup; "Medium" corresponds to a three-month notice period and a two-year lockup; and "Long" corresponds to a six-month notice period and a three-year lockup. ${ }^{6}$ Costs are estimated per $\$ 100$ of fund asset value.

Table 4 lists the $25^{\text {th }}, 50^{\text {th }}$, and $75^{\text {th }}$ percentiles of the cross-sectional distributions of restriction costs. For all levels of restrictions, the interquartile spread is economically significant, with the $75^{\text {th }}$ percentile fund's costs about double that of the costs corresponding to the $25^{\text {th }}$ percentile fund. The "Short" restrictions, for example, levy a $\$ 6.81$ cost at the $25^{\text {th }}$ percentile versus $\$ 13.51$ at the $75^{\text {th }}$ percentile. As expected, the costs are increasing in the duration of the restrictions, although the largest increase occurs when going from "Short" to "Medium" restrictions compared to the "Medium" to "Long" set of restrictions. These results indicate that restriction costs vary considerably across funds and so should be an important component of an investor's decision in fund selection.

\section{Conclusion}

We model the investor's decision to redeem capital from a hedge fund as a real option and develop a methodology to value the cost of lockups and notice periods for a risk-averse CRRA investor. An investor who is always able to redeem from the fund and receive the prevailing NAV has an unrestricted liquidity option. Lockups and notice periods are exercise restrictions that reduce the value of the liquidity option. The restriction costs are estimated by the resulting reduction in the value of the liquidity option that investors possess.

We value the liquidity options using a lattice which accounts for the possibility of early exercise and incorporates probabilities of fund failure that vary with fund age and fund performance. We find that typical parameter values can generate a one-time cost of $4 \%$ of initial NAV for a two-year lockup and a three-month notice period. When fund managers can unilaterally suspend an investor's real option to redeem, the reduction in hedge fund value is equivalent to an expected return shortfall of over $5 \%$ per year assuming that an investor initially values the fund investment at par. This result suggests that hedge fund investors should be more concerned about the discretion asserted by fund managers in their partnership agreement, and conditions under which redemption suspensions can be imposed, rather than by the standard terms of lockup and notice periods.

\footnotetext{
${ }^{6}$ An alternative is to use each fund's stated lockup and notice period. We decided to value several packages of restrictions because the restriction information is available only for a small subset of funds. In addition, in the wake of the financial crisis, many funds are revising their redemption restrictions and they may be negotiable for some investors.
} 


\section{Appendix}

\section{A.1. Hedge fund value with no liquidity option}

Let $H_{t, j}$ denote the value of a hedge fund per share conditional on survival at node $(t, j)$ from the perspective of a "passive" investor who does not possess a liquidity option. This is the case where a fund manager can unilaterally impose restrictions on exercise that reduce the value of an investor's liquidity option, and in the extreme case can eliminate the liquidity option completely. In other words, we can interpret $H_{t, j}$ as the extreme case when a hedge fund manager disables an investor's liquidity option.

A passive investor will receive a payoff at the time of fund failure or at date $T$ if the fund survives until the end of the investment horizon. For simplicity, we assume that if the fund fails, the investor places the proceeds in a risk-free account until time $T$. Assume that at the terminal set of nodes investors receive the NAV, $S_{T, j}$.

Prior to the terminal set of nodes, the value of the investment conditional on survival is given by its certainty equivalent, which is defined as the current value of a risk-free security that matures at time $T$ and provides a guaranteed level of utility equal to the expected utility of holding the hedge fund. At node $(T-1, j)$, the value of the fund, $H_{T-1, j}$, is the solution to

$$
\frac{1}{1-\gamma}\left(R_{f} H_{T-1, j}\right)^{1-\gamma}=\frac{1}{1-\gamma}\left[\pi_{T-1, j}\left(S_{T-1, j} l\right)^{1-\gamma}+\left(1-\pi_{T-1, j}\right)\left(p S_{T, j}^{1-\gamma}+(1-p) S_{T, j+1}^{1-\gamma}\right)\right],
$$

where $R_{f}$ is the gross risk-free return for a period of length $\Delta t$.

The RHS of equation (A1) is the expected utility of random time $T$ wealth computed at node $(T-1, j)$ when the NAV of the fund is $S_{T-1, j}$. The first term in the square brackets allows for the probability that the fund fails between $T-1$ and $T$, which results in a loss due to the liquidation cost and a payoff at $T$ equal to $S_{T-1, j} l$. The second term captures the expected utility of an investment in the fund if failure does not occur. The LHS of equation (A1) is the utility of a certain time $T$ wealth level $R_{f} H_{T-1, j}$, which

gives the same expected utility of holding the hedge fund. We use $H_{T-1, j}$ to express the certainty equivalent of the hedge fund investment at node $(T-1, j)$, so that the investor is indifferent between staying in the fund at node $(T-1, j)$ and exchanging for cash equal to $H_{T-1, j}$, which is then deposited in a risk-free account. We solve for $H_{T-1, j}$ by rearranging (A1).

Prior to $T-1$, the computation is similar to (A1) but involves next-period certainty equivalents:

$$
H_{t, j}=R_{f}^{-1}\left[\pi_{t, j}\left(S_{t, j} l\right)^{1-\gamma}+\left(1-\pi_{t, j}\right)\left(p H_{t+1, j}^{1-\gamma}+(1-p) H_{t+1, j+1}^{1-\gamma}\right)\right]^{1 / 1-\gamma} .
$$


Under CRRA utility, the indirect utility is also of the power form, which permits using the certainty equivalent in (A1) in the computation of equation (A2). The recursion in (A2) is repeated until the initial node in the lattice is reached, where the value of the hedge fund to the investor is $H_{0,1}$.

The case of risk neutrality can be represented by setting $\gamma=0$. For a risk-neutral investor, the calculations in (A1) and (A2) simply compute the discounted, expected payoff, where the discounting occurs at the risk-free rate. In general risk-neutral valuation is not possible because the underlying asset, the hedge fund, cannot be shorted. Thus, valuation by utility methods must be used.

\section{A.2. Hedge fund value with unrestricted liquidity option}

Let $O_{t, j}$ denote the value of the hedge fund per share at node $(t, j)$, again conditional on survival, but this time from the perspective of an "active" investor who possesses an unrestricted liquidity option. The investor will exercise the option at a given node $(t, j)$ if an immediate payoff equal to the fund NAV, $S_{t, j}$, provides greater utility than the expected utility of remaining in the fund.

As before, we assume that the investor receives the NAV at the terminal set of nodes. Prior to the terminal set of nodes, the hedge fund value is the maximum of immediate exercise of the redemption option and the certainty equivalent. At node $(T-1, j)$, the value of the fund, $O_{T-1, j}$, is the solution to

$$
O_{T-1, j}=\max \left(S_{T-1, j}, R_{f}^{-1}\left[\pi_{T-1, j}\left(S_{T-1, j} l\right)^{1-\gamma}+\left(1-\pi_{T-1, j}\right)\left(p S_{T, j}^{1-\gamma}+(1-p) S_{T, j+1}^{1-\gamma}\right)\right]^{1 / 1-\gamma}\right) .
$$

The first element in the RHS of equation (A3) is the value received by the investor upon immediate exercise, which is the NAV of the fund at the beginning of the node, $S_{T-1, j}$. The second term is the value of the fund to the investor expressed as a certainty equivalent computed at node $(T-1, j)$. If the investor obtains a higher utility by receiving the NAV, the investor exercises his option. Prior to time $T-1$, the option value is computed as

$$
O_{t, j}=\max \left(S_{t, j}, R_{f}^{-1}\left[\pi_{t, j}\left(S_{t, j} l\right)^{1-\gamma}+\left(1-\pi_{t, j}\right)\left(p O_{t+1, j}^{1-\gamma}+(1-p) O_{t+1, j+1}^{1-\gamma}\right)\right]^{1 / 1-\gamma}\right) .
$$

The initial value of the hedge fund to the investor with an unrestricted liquidity option is therefore $O_{0,1}$ and the initial value of the liquidity option is $O_{0,1}-H_{0,1}$.

\section{A.3. Valuing a lockup}

A lockup prevents an investor from exercising his liquidity option prior to date $L$. Let $O_{L, t, j}$ denote the value of a hedge fund, at node $(t, j)$, with a liquidity option restricted by a lockup. After the lockup expires, there is no restriction, hence $O_{L, t, j}=O_{t, j}$ for $t>L$, and hedge fund values can be computed as described in Section A.2. For $t \leq L$, however, the investor cannot exercise the liquidity option, hence 


$$
O_{L, t, j}=R_{f}^{-1}\left[\pi_{t, j}\left(S_{t, j} l\right)^{1-\gamma}+\left(1-\pi_{t, j}\right)\left(p O_{L, t+1, j}^{1-\gamma}+(1-p) O_{L, t+1, j+1}^{1-\gamma}\right)\right]^{1 / 1-\gamma}
$$

for $t \leq L$.

The initial value of a hedge fund with a liquidity option subject to a lockup is therefore $O_{L, 0,1}$ and the initial value of the restricted liquidity option is $O_{L, 0,1}-H_{0,1}$. The ex-ante cost of the lockup itself is the difference between the unrestricted and restricted options, i.e., $O_{0,1}-O_{L, 0,1}$. 


\section{References}

Ackermann, C., McEnally, R., Ravnescraft, D., 1999. The performance of hedge funds: risk, return, and incentives. Journal of Finance 54, 833-874.

Ang, A., and N. Bollen, 2010, Locked Up by a Lockup: Valuing Liquidity as a Real Option. Financial Management, forthcoming.

Aragon, G. O., 2007. Share restrictions and asset pricing: evidence from the hedge fund industry. Journal of Financial Economics 83, 33-58.

Asness, C., Krail, R., Liew, J., 2001. Do hedge funds hedge? Journal of Portfolio Management 28, 6-19.

Bollen, N. P. B., Pool, V., 2008. Conditional return smoothing in the hedge fund industry. Journal of Financial and Quantitative Analysis 43, 267-298.

Brown, S. J., Goetzmann, W. N., Park, J., 2001. Careers and survival: competition and risk in the hedge fund and CTA industry. Journal of Finance 56, 1869-1886.

Derman, E., 2007. A simple model for the expected premium for hedge fund lockups. Journal of Investment Management 5, 5-15.

Derman, E., Park, K. S., Whitt, W., 2007. Markov chain models to estimate the premium for extended hedge fund lockups. Working paper, Columbia University.

Ding, B., Getmansky, M., Liang, B., Wermers, R., 2007. Investor flows and share restrictions in the hedge fund industry. Working paper, University of Massachusetts.

Getmansky, M., Lo, A. W., Makarov, I., 2004. An econometric model of serial correlation and illiquidity in hedge fund returns. Journal of Financial Economics 74, 29-609.

Grecu, A., Malkiel, B. G., Saha, A., 2006. Why do hedge funds sop reporting their performance? CEPS working paper 124.

Jagannathan, R., Malakhov, A., Novikov, D., 2006. Do hot hands persist among hedge fund managers? An empirical evaluation. NBER working paper 12015.

Lerner, J., Schoar, A., 2004. The illiquidity puzzle: theory and evidence from private equity. Journal of Financial Economics 72, 3-40.

Liang, B., 2000. Hedge funds: the living and the dead. Journal of Financial and Quantitative Analysis 35, 309-326.

Ramadorai, T., 2008. The secondary market for hedge funds and the closed-hedge fund premium. University of Oxford working paper. 


\section{Table 1. Hedge Fund Failure Process Parameters.}

Listed are parameter estimates obtained by maximum likelihood for the following hazard rate to model the probability of hedge fund failure:

$$
\lambda(t ; z)=\lambda q(\lambda t)^{q-1} /\left[1+(\lambda t)^{q}\right] \exp (z \beta)
$$

where $\lambda, q$, and $\beta$ are parameters, $t$ is the age of the fund, and $z$ is the value of a performance score which equals the number of cross-sectional standard deviations the fund's cumulative return is from the crosssectional mean.

\begin{tabular}{lrrr} 
& $\lambda$ & $q$ & $\beta$ \\
\cline { 2 - 4 } Estimate & 0.0164 & 3.1539 & -0.2302 \\
Std Error & 0.0001 & 0.0217 & 0.0057 \\
\hline
\end{tabular}




\section{Table 2. Combined Cost of Lockup and Notice Period.}

Listed are the combined costs of lockups and notice periods of different lengths, in months, per share of a two-year old hedge fund with initial NAV of $\$ 100$. Returns are normally distributed with annual volatility $15 \%$ and expected return of $10 \%$. Fund failures arrive randomly following a log-logistic distribution. Upon failure, NAV drops $25 \%$ and the investor receives the remaining assets as a liquidating dividend. The investor has risk aversion parameter $\gamma=3$.

\begin{tabular}{rrrrrr} 
& \multicolumn{5}{c}{ Lockup } \\
\cline { 2 - 6 } Notice & 12 & 24 & 36 & 48 & 60 \\
\hline 1 & 0.67 & 3.51 & 6.62 & 8.93 & 10.40 \\
2 & 0.84 & 3.78 & 6.85 & 9.09 & 10.49 \\
3 & 1.03 & 4.06 & 7.07 & 9.23 & 10.58 \\
4 & 1.24 & 4.33 & 7.29 & 9.37 & 10.66 \\
5 & 1.46 & 4.60 & 7.49 & 9.51 & 10.74 \\
\hline
\end{tabular}




\section{Table 3. Incremental Required Returns.}

Listed are incremental returns required to offset the cost of liquidity restrictions in a two-year old hedge fund with initial NAV of $\$ 100$ and a ten-year life. Base-case returns are normally distributed with expected return as listed. Annual volatility is adjusted so that the value to an investor with an unrestricted liquidity option is equal to the initial NAV. Fund failures arrive randomly following a log-logistic distribution. Upon failure, NAV drops $25 \%$ and the investor receives the remaining assets as a liquidating dividend. The investor has risk aversion parameter $\gamma=3$. The four columns are the additional expected return required to offset restrictions for: an investor with no liquidity option, $H_{0,1}$; an investor with a liquidity option subject to a two-year lockup and a three-month notice period, $O_{L N P, 0,1}$; an investor with a liquidity option subject only to a lockup, $O_{L, 0,1}$; and an investor with a liquidity option subject only to a notice period, $O_{N P, 0,1}$.

\begin{tabular}{rrrrrr}
$\mu$ & $\sigma$ & $H_{0,1}$ & $O_{L N P, 0,1}$ & $O_{L, 0,1}$ & $O_{N P, 0,1}$ \\
\hline $10.0 \%$ & $17.9 \%$ & $5.58 \%$ & $3.53 \%$ & $3.20 \%$ & $0.28 \%$ \\
$11.0 \%$ & $19.7 \%$ & $5.62 \%$ & $3.56 \%$ & $3.23 \%$ & $0.28 \%$ \\
$12.0 \%$ & $21.3 \%$ & $5.66 \%$ & $3.59 \%$ & $3.25 \%$ & $0.28 \%$ \\
$13.0 \%$ & $22.9 \%$ & $5.70 \%$ & $3.62 \%$ & $3.28 \%$ & $0.28 \%$ \\
$14.0 \%$ & $24.3 \%$ & $5.74 \%$ & $3.65 \%$ & $3.31 \%$ & $0.29 \%$ \\
$15.0 \%$ & $25.7 \%$ & $5.78 \%$ & $3.67 \%$ & $3.33 \%$ & $0.29 \%$ \\
$16.0 \%$ & $26.9 \%$ & $5.82 \%$ & $3.70 \%$ & $3.36 \%$ & $0.29 \%$ \\
$17.0 \%$ & $28.2 \%$ & $5.86 \%$ & $3.73 \%$ & $3.38 \%$ & $0.29 \%$ \\
$18.0 \%$ & $29.4 \%$ & $5.90 \%$ & $3.76 \%$ & $3.41 \%$ & $0.29 \%$ \\
\hline
\end{tabular}


Table 4. Actual Restriction Costs January 2009.

Listed are the $25^{\text {th }}, 50^{\text {th }}$, and $75^{\text {th }}$ percentiles of the cross-sectional distribution of combined costs of lockups and notice periods per share of live hedge funds in the CISDM/TASS database with initial NAV scaled to $\$ 100$. "Short" corresponds to a one-month notice period and a one-year lockup; "Medium" corresponds to a three-month notice period and a two-year lockup; and "Long" corresponds to a six-month notice period and a three-year lockup. Costs are estimated at the beginning of 2009 separately for each fund using the fund's age at that time. Future returns are assumed normally distributed with annual volatility and expected return estimated for each fund using their historical returns. Fund failures arrive randomly following a log-logistic distribution. Upon failure, NAV drops $25 \%$ and the investor receives the remaining assets as a liquidating dividend. The investor has risk aversion parameter $\gamma=3$.

\begin{tabular}{lrrr} 
& \multicolumn{3}{c}{ Restrictions } \\
\cline { 2 - 4 } Percentile & Short & Medium & Long \\
\hline $25^{\text {th }}$ & 6.81 & 10.59 & 12.56 \\
$50^{\text {th }}$ & 10.21 & 15.68 & 18.55 \\
$75^{\text {th }}$ & 13.51 & 20.94 & 25.20 \\
\hline
\end{tabular}




\section{Figure 1. Fitted vs. Actual Number of Hedge Fund Failures.}

Depicted by hollow squares is the number of defunct hedge funds in the CISDM/TASS hedge fund database with lifespan equal to the values on the horizontal axis. Depicted by the solid line is the predicted number of hedge funds with lifespan equal to the values on the horizontal axis.

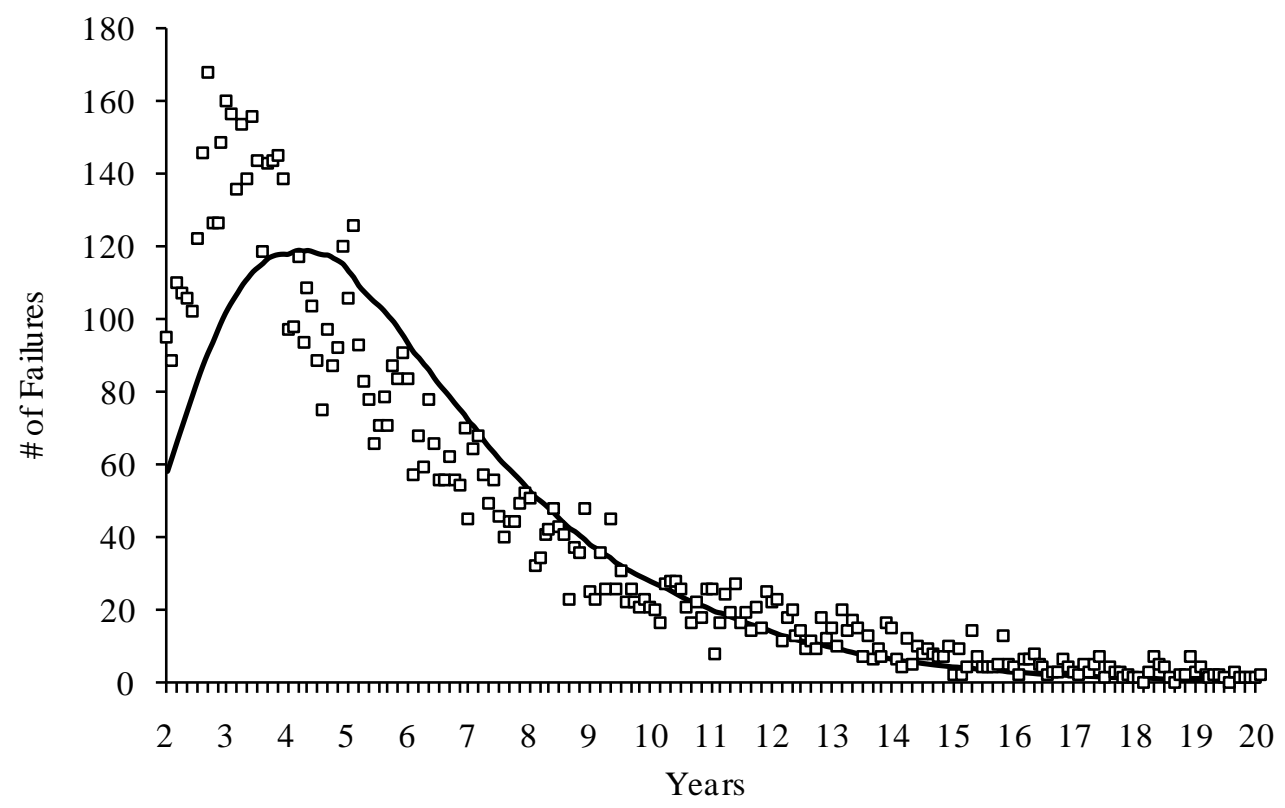

